\title{
NEW PHYSICS POTENTIAL WITH A NEUTRINO TELESCOPE
}

\author{
N. Arteaga-Romero ${ }^{a}$, C. Carimalo ${ }^{a}$ \\ A. Nicolaidis ${ }^{b}$, O. Panella ${ }^{c}$ and G. Tsirigoti ${ }^{b *}$ \\ (a) Laboratoire de Physique Corpusculaire, Collège de France, \\ 11 Pl. Marcelin Berthelot F-75231, Paris Cedex 05, France \\ (b) Department of Theoretical Physics, University of Thessaloniki, GR-54006 Thessaloniki, Greece \\ (c) INFN, Sezione di Perugia, Via A. Pascoli, I-06123 Perugia, Italy
}

\begin{abstract}
Active Galactic Nuclei are considered as sources of neutrinos, with neutrino energies extending up to $10^{18} \mathrm{eV}$. It is expected that these highly energetic cosmic neutrinos will be detected by the neutrino telescopes, presently under construction. The detection process is very sensitive to the total $\nu_{\mu} N$ crosssection. We examine how $\sigma_{t o t}\left(\nu_{\mu} N\right)$ changes at high energies, by the single production of excited fermions $\left(\mu^{*}, \nu_{\mu}^{*}\right)$. For parameters (masses, couplings) of the excited fermions allowed by the experimental constraints, we find that for energies of the incoming $\nu_{\mu}$ above $100 \mathrm{TeV}$ the cross-section for single production of $\mu^{*}, \nu_{\mu}^{*}$ supersedes the standard total cross-section.

Keywords : Cosmic neutrinos, Galaxy nuclei, Neutrino reactions. PACS number(s) : 13.15.-f; 13.85.Tp
\end{abstract}

Typeset using REVTEX

*e-mail addresses : carimalo@in2p3.fr, nicolaid@ccf.auth.gr, o.panella@perugia.infn.it 
High energy is the prerequisite for the study of matter in shorter distances, or the production of new massive states. It appears that the most powerful accelerators are the cosmic accelerators in the outer space. Active Galactic Nuclei (hereafter AGN) are the most powerful radiation sources known in the Universe and they have long been considered as prodigious particle accelerators and beam dumps [1] [3]. As the term indicates, AGN are the central regions of certain galaxies in which the emission of radiation can rival or even surpass the total power output of the entire galaxy by as much as a thousand fold. All this power is emitted from a region which is extremely small by galactic standards. Typical AGN luminosities are in the range $10^{44} \mathrm{erg} / \mathrm{s}$ to $10^{47} \mathrm{erg} / \mathrm{s}$. The tremendous power output suggests that the source that powers AGN is gravity, i.e. matter accretion into a supermassive black hole located at the center of the galaxy. Within AGN, particles and in particular protons can be accelerated to very high energies. The maximum energy $E_{\max }$ attained by protons is determined by balancing the proton acceleration rate with the proton energy loss rate. Simple estimates indicate that $E_{\max } \sim 10^{18} \mathrm{eV}$. The relativistic protons may interact with matter or radiation in the AGN to produce pions whose decay products include photons and neutrinos. It is expected that neutrinos and photons are equally abundant and their spectra should be in general of the same form as the parent proton spectrum. AGN photons have been already observed by the EGRET instrument [4] aboard the Compton Gamma-Ray Observatory and the Whipple Observatory [5]. While photons are subject to photon-photon absorption within the volume of AGN (whose optical depth is proportional to the photon energy, thus cutting off the highest energy photons), neutrinos suffer no such absorption, indicating that AGN could be the most luminous high energy neutrino sources in the Universe. The diffuse isotropic neutrino flux from all AGN has been estimated [6, []] and it might be observable by the neutrino telescopes, presently under construction.

Cosmic high energy muon neutrinos can be observed by detecting the long range muons produced in charged current muon neutrino-nucleon interactions. The effective detector volume is enhanced in proportion to the range of the produced muon (typically several kilometers). At high energies (above $1 \mathrm{TeV}$ ) the produced muon is almost aligned to the 
parent muon neutrino, thus knowledge of the muon direction fixes the origin of the neutrino in the sky. Neutrino telescopes [3, 8] will provide then a unique window to our cosmos. In addition to opening new territory in astronomy, neutrino telescopes may help, because of the enormous energies of the particle interactions involved, to study fundamental physics in the ultrahigh energy (UHE) regime. Specific signatures of "new physics" at a neutrino telescope have been analyzed already. These include the search for substructure of the elementery particles [9], multiple production of gauge bosons [10,11], scalar leptoquarks [12 and contact four-fermion interactions [11]. Detection of UHE cosmic neutrinos depends crucially upon the total $\nu_{\mu} N$ cross-section, $\sigma_{\text {tot }}(E)$. To reduce background, at the detection site one looks for upward moving muons, induced by neutrinos coming from the other side of the Earth. At energies above a few $\mathrm{TeV}$ the Earth starts becoming opaque to neutrinos. The neutrino propagation inside the Earth has been studied in ref. 13. At very high energies and for a neutrino energy spectrum which is not flat, the neutrino attenuation can be approximated by the simple absorption formula

$$
I(E, \tau) \simeq I_{0}(E) \exp \left[-\sigma_{t o t}(E) \tau\right]
$$

In the above expression $\tau$ is the total number of nucleons per unit area encountered by the neutrino along its path through the Earth and $I_{0}$ is the initial neutrino intensity. For a neutrino going through the center of the Earth $\tau=\tau_{\max } \simeq 6 \times 10^{33} \mathrm{~cm}^{-2}$. Neutrino absorption is very sensitive to $\sigma_{\text {tot }}(E)$. Any sort of new physics (new interaction terms, production of new massive states) will increase $\sigma_{\text {tot }}(E)$ with a subsequent dramatic reduction of the neutrino intensity at the detection site.

In this Letter we address the implications of a composite scenario as regards the detection of AGN neutrinos. The idea that at an energy scale $\Lambda_{\mathrm{C}}$ quarks and leptons might show an internal structure has been around for quite some time [14]. Various models describing quarks and leptons in terms of preon bound states have been proposed, but so far no consistent dynamical composite theory has been found [15]. However a natural consequence of this scenario is the existence of excited states of the ordinary fermions with masses at least 
of the order of the compositeness scale. Effective couplings between the excited and light leptons have been proposed, using weak isospin $\left(I_{W}\right)$ and hyper-charge $(Y)$ conservation. Within this model, it is assumed that the lightness of the ordinary leptons could be related to some global unbroken chiral symmetry which would produce massless bound states of preons in the absence of weak perturbations due to $S U(2) \times U(1)$ gauge and Higgs interactions. The large mass of the excited leptons arises from the unknown underlying dynamics and not from the Higgs mechanism. We restrict ourselves to one family and consider spin-1/2 excited states grouped in multiplets with $I_{W}=1 / 2$ and $Y=-1$,

$$
L=\left(\begin{array}{c}
\nu_{\mu}^{*} \\
\mu^{*}
\end{array}\right)
$$

which can couple to the light left-handed multiplet

$$
\ell_{L}=\frac{1-\gamma_{5}}{2}\left(\begin{array}{c}
\nu_{\mu} \\
\mu
\end{array}\right)
$$

through the gauge fields $\vec{W}^{\mu}$ and $B^{\mu}$, the relevant interaction (of magnetic type) being written [16] in terms of two new independent coupling constants $f$ and $f^{\prime}$, as

$$
\begin{aligned}
\mathcal{L}_{i n t}=\frac{g f}{\Lambda_{\mathrm{C}}} \bar{L} \sigma_{\mu \nu} & \frac{\vec{\tau}}{2} \ell_{L} \cdot \partial^{\nu} \vec{W}^{\mu} \\
& +\frac{g^{\prime} f^{\prime}}{\Lambda_{\mathrm{C}}}\left(-\frac{1}{2} \bar{L} \sigma_{\mu \nu} \ell_{L}\right) \cdot \partial^{\nu} B^{\mu}+\text { h.c. }
\end{aligned}
$$

where $\vec{\tau}$ are the Pauli $S U(2)$ matrices, $g$ and $g^{\prime}$ are the usual $S U(2)$ and $U(1)$ gauge coupling constants, and the factor of $-1 / 2$ in the second term is the hyper-charge of the $U(1)$ current. This effective Lagrangian has been widely used in the literature to predict production cross sections and decay rates of the excited particles at colliders [16]. The extension to quarks and strong interactions as well as to other multiplets and a detailed discussion of the spectroscopy of the excited particles can also be found in [16], while for a review of compositeness phenomenology we refer to ref. [17]. The effective interaction, written out in terms of the physical gauge fields is

$$
\mathcal{L}_{e f f}=\sum_{V=\gamma, Z, W} \frac{e}{\Lambda_{c}} C_{V \ell L} \bar{L} \sigma^{\mu \nu}\left(1-\gamma_{5}\right) \ell \partial_{\mu} V_{\nu}+h . c .
$$


to be compared with the standard model interaction

$$
\mathcal{L}_{S M}=\sum_{V=\gamma, Z, W} e \bar{\ell}^{\prime} \gamma^{\mu}\left(A_{V \ell^{\prime} \ell}-B_{V \ell^{\prime} \ell} \gamma_{5}\right) \ell V_{\mu}+\text { h.c. }
$$

The relevant effective couplings are

$$
\begin{array}{r}
C_{W \nu \mu^{*}}=\frac{f}{2 \sqrt{2} \sin \theta_{W}} \\
C_{Z \nu \nu^{*}}=\frac{f \cot \theta_{W}+f^{\prime} \tan \theta_{W}}{4} \\
C_{\gamma \nu \nu^{*}}=\frac{f-f^{\prime}}{4}
\end{array}
$$

For the Weinberg angle, we have used $\sin ^{2} \theta_{W}=0.226$. In the following we study production of excited leptons in the collisions of UHE neutrinos with nucleons

$$
\begin{gathered}
\nu_{\mu} N \rightarrow \nu_{\mu}^{*} X \\
\nu_{\mu} N \rightarrow \mu^{*} X
\end{gathered}
$$

For the partonic process $\nu_{\mu} q(\bar{q}) \rightarrow \nu_{\mu}^{*}\left(\mu^{*}\right) q^{\prime}$ we find

$$
\begin{aligned}
\frac{d \hat{\sigma}}{d Q^{2}}= & \frac{2 \pi \alpha^{2}}{\hat{s}^{2} \Lambda_{\mathrm{C}}{ }^{2}} Q^{2} \sum_{V, V^{\prime}} \frac{1}{\left(Q^{2}+M_{V}^{2}\right)\left(Q^{2}+M_{V^{\prime}}^{2}\right)} \times \\
& \left\{D_{V V^{\prime}}\left[2 \hat{s}^{2}-\left(2 \hat{s}-m_{*}^{2}\right)\left(m_{*}^{2}+Q^{2}\right)\right] \pm E_{V V^{\prime}} m_{*}^{2}\left(2 \hat{s}-m_{*}^{2}-Q^{2}\right)\right\}
\end{aligned}
$$

where $m^{*}$ is the mass of the produced excited lepton and the \pm sign depends on whether the neutrino scatters off a quark or an antiquark. The sum over $V$ restricts only to $W$ for the charged current process but includes both $\gamma$ and $Z$ for the neutral current process (see ref. [18] for calculations of excited lepton production in $e^{+} e^{-}$and ep colliders). We have also defined

$$
\begin{gathered}
D_{V V^{\prime}}=4 C_{V \nu \nu^{*}\left(\mu^{*}\right)} C_{V^{\prime} \nu \nu^{*}\left(\mu^{*}\right)}\left(A_{V q q^{\prime}} A_{V^{\prime} q q^{\prime}}+B_{V q q^{\prime}} B_{V^{\prime} q q^{\prime}}\right) \\
E_{V V^{\prime}}=4 C_{V \nu \nu^{*}\left(\mu^{*}\right)} C_{V^{\prime} \nu \nu^{*}\left(\mu^{*}\right)}\left(A_{V q q^{\prime}} B_{V^{\prime} q q^{\prime}}+B_{V q q^{\prime}} A_{V^{\prime} q q^{\prime}}\right)
\end{gathered}
$$


The hadronic cross-section is related to the partonic one by the usual convolution with the parton distribution functions

$$
\frac{d \sigma}{d x d Q^{2}}\left(\nu_{\mu} N \rightarrow \nu^{*}\left(\mu^{*}\right) X\right)=\sum_{q} \frac{d \hat{\sigma}}{d Q^{2}}\left(\nu_{\mu} q \rightarrow \nu^{*}\left(\mu^{*}\right) q^{\prime}\right) f_{q}\left(x, Q^{2}\right)
$$

A cutoff $Q_{0}$ is introduced in the $Q^{2}$ integration to avoid the region in which perturbative QCD is not valid.t The integrated cross section is given by

$$
\sigma=\int_{\left(m_{*}^{2}+Q_{0}^{2}\right) / S}^{1} d x \int_{Q_{0}^{2}}^{\hat{s}-m_{*}^{2}} d Q^{2} \frac{d \sigma}{d x d Q^{2}}
$$

where $\hat{s}=x S$ and $S=2 M_{N} E_{\nu}$ as usual. The parameters $f / \Lambda_{c}, f^{\prime} / \Lambda_{c}$ and $m^{*}$ are already constrained by unsuccesful searches for excited leptons at colliders and accelerators [19]. More severely constrained are the parameters referring to the first family of excited leptons $e^{*}, \nu_{e}^{*}$. As regards the parameters corresponding to $\mu^{*}, \nu_{\mu}^{*}$, for the purpose of numerical calculations we have chosen two illustrative sets :

$$
\begin{gathered}
\text { (i) } \frac{f}{\Lambda_{c}}=\frac{f^{\prime}}{\Lambda_{c}}=0.03 \mathrm{GeV}^{-1} \quad m_{*}=130 \mathrm{GeV} . \\
\text { (ii) } f=0 \quad \frac{f^{\prime}}{\Lambda_{c}}=0.03 \mathrm{GeV}^{-1} \quad m_{*}=130 \mathrm{GeV} .
\end{gathered}
$$

Both sets of parameters are the upper bounds suggested by the experimental information [19]. For the parton distribution functions of an isoscalar nucleon we used the GRV parametrization [20]. Fig.1 shows $\sigma_{s m}$, the total $\nu_{\mu} N$ cross-section as given by the standard model (solid line). In the energy range considered the standard model prediction is relatively safe. At higher energy, the nucleon is probed at very small $x$ values, where BFKL physics [21] might be operative. The precise rise with the energy of $\sigma_{s m}$ has been studied recently [22]. In the same fig.1, we show the extra contribution to $\sigma_{t o t}\left(\nu_{\mu} N\right)$, if excited leptons are

\footnotetext{
${ }^{1}$ For $f \neq f^{\prime}$ and very small values of $Q^{2}$, coherent and incoherent elastic processes should contribute, thus enhancing further the cross-section. We do not consider, in this first explorative work, these contributions.
} 
produced. In the case $f=f^{\prime}$ the excited leptons $\mu^{*}, \nu_{\mu}^{*}$ are produced via $\mathrm{W}$ and $\mathrm{Z}$ exchange (dotted line). In the case $f=0$, there is no transition coupling to $\mathrm{W}$ and only the $\nu_{\mu}^{*}$ is produced, via $\mathrm{Z}$ and $\gamma$ exchange (dashed line). Whenever there is a significant coupling of $\nu_{\mu}$ to $\nu_{\mu}^{*}$ via a photon exchange, i.e. $f \neq f^{\prime}$ (see eq. 7 ), the photon propagator dominates over the corresponding propagators of massive gauge bosons at low momentum transfer, with a resulting enhanced cross-section near threshold. We observe that, for $f=f^{\prime}$ and with the assumed values for the couplings and the masses of the excited leptons, the cross section for the production of excited leptons supersedes the standard cross-section for $\nu_{\mu}$ energies around $100 \mathrm{TeV}$.

At the energies considered $\left(E_{\nu}>10 \mathrm{TeV}\right)$ all phenomenological models [6] indicate that the diffuse isotropic neutrino flux from all AGN dominates over the flux of atmospheric neutrinos. Therefore any anisotropy of the measured neutrino flux at the detection site should be attributed to the neutrino attenuation inside the Earth. The shadowing of UHE neutrinos by the Earth involves the weak charged current, resulting into absorption, and the weak neutral current, which provides a redistribution of the neutrino energy [13]. For our purposes, we use the rough estimate provided by eq.(1). For neutrinos scratching the Earth, $\tau$ is very small and we have access to the initial neutrino intensity $I_{0}(E)$. For neutrinos incident at a different angle and traversing the Earth, we obtain information about $I(E, \tau)$. Therefore the ratio $I(E, \tau) / I_{0}(E)$ is experimentally accessible. The knowledge of the density of the Earth through the seismic data [23] allows one to extract $\sigma_{\text {tot }}(E)$ at high energy from the absorption factor. Fig. 2 shows the absorption factor for $\tau=2 \times 10^{33} \mathrm{~cm}^{-2}$ with $\sigma_{t o t}=\sigma_{s m}$ and with $\sigma_{\text {tot }}=\sigma_{s m}+\sigma_{\text {new }}$, where $\sigma_{\text {new }}$ originates from the production of excited leptons.

In summary we analyzed the possibility of unravelling new physics in the collisions of UHE cosmic neutrinos with nucleons. The energy domain reached is higher than the present HERA energy region and the muonic sector (rather than the electronic) is explored. We focused our attention unto the neutrino absorption factor, which is sensitive to the total $\nu_{\mu} N$ cross-section. In order to specify further the origin of the new physics, specific signatures are needed. In our case, multiple muons or electromagnetic showers will indicate the production 
of $\mu^{*}, \nu_{\mu}^{*}$. Work along these lines is in progress.

\section{ACKNOWLEDGMENTS}

This work was partially supported by the EU program "Human Capital and Mobility", under contract No. CHRX-CT94-0450. The authors wish to acknowledge useful discussions with the members of the Theory group of the Laboratoire de Physique Corpusculaire at Collège de France. 


\section{REFERENCES}

[1] I. Robson, Active Galactic Nuclei, Willey-Praxis series in Astronomy and Astrophysics (1996).

[2] D. Kazanas in NESTOR, 3rd NESTOR International Workshop, Pylos 1993, edited by L. Resvanis, p. 29.

[3] T. Gaisser, F. Halzen and T. Stanev, Phys. Rep. 258 (1995) 173.

[4] C. Fichtel et al., Astrophys. J. Suppl. 94 (1994) 551.

[5] M. Punch et al., Nature 160 (1992) 477.

A. Kerrick et al., Astrophys. J. 438 (1995) L59.

J. Quinn et al., IAU Circular 6169 (June 16, 1995).

[6] F. Stecker et al., Phys. Rev. Lett. 66 (1991) 2697 ; ibid 69 (1992) 2738 (E)

F. Stecker and M. Salamon, High Energy Neutrinos from Quasars, NASA preprint (astro-ph/9501064).

[7] L. Nellen, K. Mannheim and P. Biermann, Phys. Rev. D 47 (1993) 5270.

[8] For the Baikal neutrino telescope see R. Wischewski, in the 3rd NESTOR International Workshop, Pylos 1993, edited by L. Resvanis, p. 213.

For the NESTOR neutrino telescope in the Mediterranean, off the coast of Pylos, see L. Resvanis in the 3rd NESTOR International Workshop, Pylos 1993, edited by L. Resvanis, p. 1.

For AMANDA, a neutrino telescope in the Antarctic ice, see P.Askebjer, Nucl. Phys. B38 (Proc. Suppl.) (1995) 287.

[9] G. Domokos and S. Kovesi-Domokos, in NESTOR, 2nd NESTOR International Workshop, Pylos, 1992, edited by L. Resvanis, p. 41.

[10] L. Dell'Agnello et al., in NESTOR, 2nd NESTOR International Workshop, Pylos, 1992, 
edited by L. Resvanis, p. 75.

[11] D. Morris and A. Ringwald, in NESTOR , 3rd NESTOR International Workshop, Pylos, 1993, edited by L. Resvanis, p. 101.

[12] L. Bergström, R. Liotta and H. Rubinstein, Phys. Lett. B 276 (1992) 231.

[13] A. Nicolaidis and A. Taramopoulos, Phys. Lett. B 386 (1996) 211.

[14] For reviews on substructure in the fermionic sector, see: M. Peskin, Proc. of the 10th Int. Symp. on Lepton-Photon Interactions at High-Energies, Bonn, August 1981; H. Terazawa, Proc. of the Europhysics Topical Conference on Flavor Mixing in Weak Interactions, Erice Italy, March 1984; W. Buchmüller, Lectures given at the 24th Int. Wochenuniversität für Kernphysik, Schladmig Austria, Feb. 1985; R. Peccei, Proc. of the Lake Louise Winter Institute, Lake Louise Canada, Feb. 1987; F. Boudjema, Int. J. Mod. Phys. A 6 (1991) 1.

[15] H. Fritzsch, G. Mandelbaum, Phys. Lett. B 102 (1981) 319; R. Barbieri, R. N. Mohapatra, A. Masiero, ibid B 105 (1981) 369.

For further references see for example: H. Harari, Phys. Rep. 104 (1984) 159.

[16] F. Low, Phys. Rev. Lett. 14 (1965) 238; N. Cabibbo, L. Maiani and Y. Srivastava, Phys. Lett. B 139 (1984) 459; F.M. Renard, Phys. Lett. B 116 (1982) 269; A. De Rujula, L. Maiani and R. Petronzio, Phys. Lett. B 140 (1984) 253; J. Kühn, H. D. Tholl and P. M. Zerwas, Phys. Lett. B 158 (1985) 270; F. Boudjema and A. Djouadi, Phys. Lett. B 240 (1990) 485; I. F. Ginzburg and D. Yu. Ivanov, Phys. Lett. B 276 (1992) 218; F. Boudjema, A. Djouadi and J. L. Kneur, Z. Phys. C 57 (1992) 425; U. Baur, I. Hinchliffe and D. Zeppenfeld, Int. J. Mod. Phys. A 2 (1987) 1285; G. Pancheri and Y. N. Srivastava, Phys. Lett. B 146 (1984) 87; P. Chiappetta and O. Panella, Phys. Lett. B 316 (1993) 368.

[17] O. Panella in the Proceedings of the International Workshop "Double Beta Decay and 
Related Topics" held at the European Centre for Theoretical Studies (ECT*), Trento, Italy, April 24-May 5, 1995. Ed. H. V. Klapdor-Kleingrothaus and S. Stoica, World Scientific, Singapore, 1996.

[18] K. Hagiwara, S. Komamiya and D. Zeppenfeld; Z. Phys. C 29 (1985) 115.

[19] The L3 Collaboration, CERN-PRE/95-190; ALEPH Collaboration, Phys. Lett. B 385 (1996) 445; OPAL Collaboration, Phys. Lett. B 386 (1996) 463.

[20] H. Plothow-Besch, "PDFLIB: Nucleon, pion, and Photon Parton Density Functions and $\alpha_{s}$ Calculations", Users's Manual - Version 6.06, W5051 PDFLIB, 1995.03.15 CERNPPE.

[21] For reviews, see L. Gribov, E. Levin and M. Ryskin, Phys. Rep. 100 (1983) 1;

E. Laenen and E. Livin, Ann. Rev. Nucl. Part. Sc. (1994) 199.

A. Martin, in : The heart of the matter, Editions Frontières, (1994) p. 157.

[22] G. Frichter, J. Ralston and D. Mackay, Phys. Rev. D 53 (1996) 1684;

R. Gandhi, C. Quigg, M. H. Reno and I. Sarcevic, Ultrahigh-energy neutrino interactions, Report to appear in Astroparticle Physics.

[23] A. Dziewonsky and D. Anderson, Phys. Earth Planet. Inter. 25 (1981) 297. 


\section{FIGURES}

FIG. 1. $\nu_{\mu} N$ cross-sections for the standard model (solid line), excited lepton production with $f=0, f^{\prime}=1$ (dashed line), with $f=f^{\prime}=1$ (dotted line).

FIG. 2. Absorption of the neutrino flux for $\tau=2 \times 10^{33} \mathrm{~cm}^{-2}$ within the standard model (solid line) compared with the larger absorption in the presence of the new physics effect: excited lepton production with $f=0, f^{\prime}=1$ (dashed line) and with $f=f^{\prime}=1$ (dotted line). 
N. Arteaga-Romero et al. ---- Figure 1

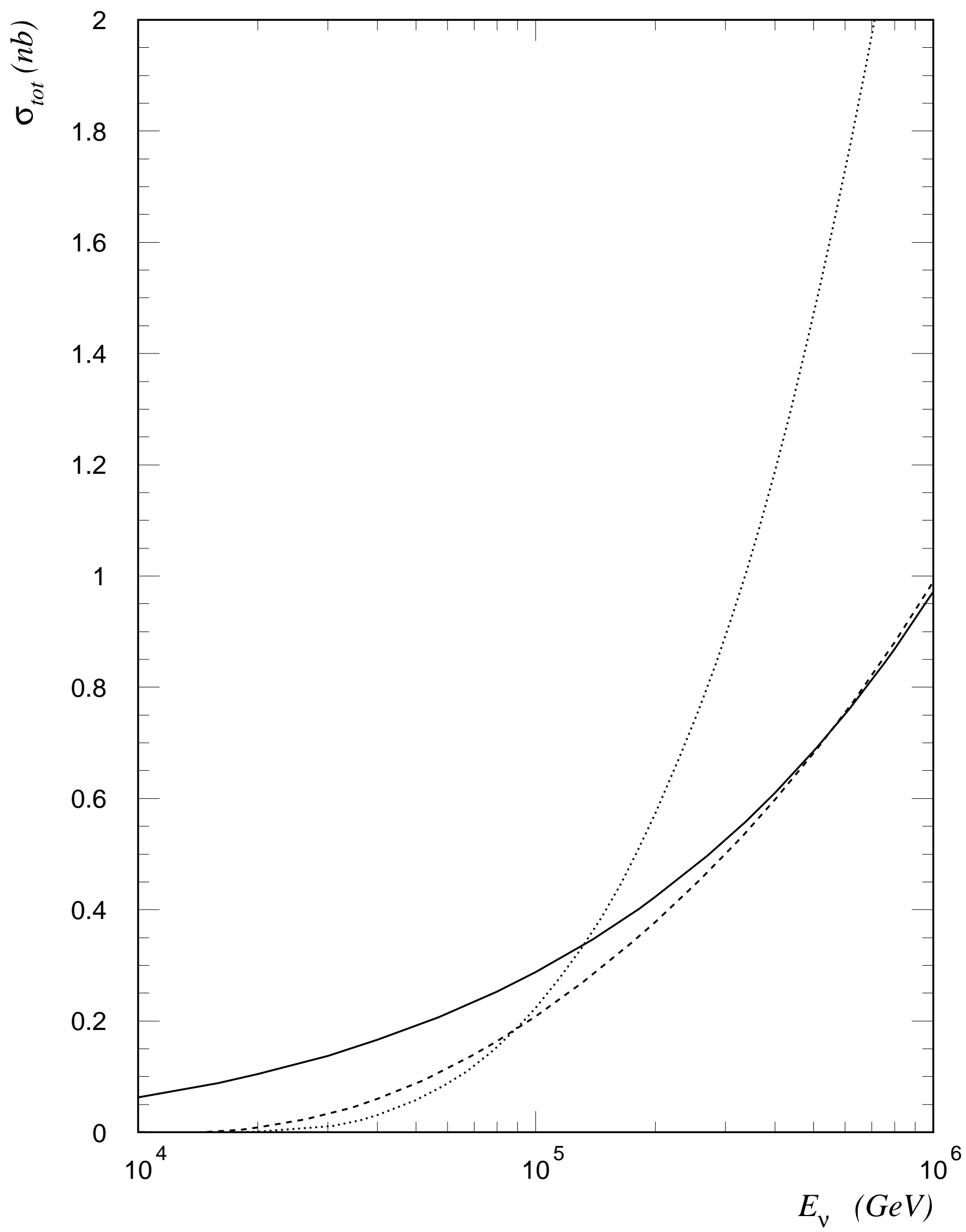


N. Arteaga-Romero et al. ---- Figure 2

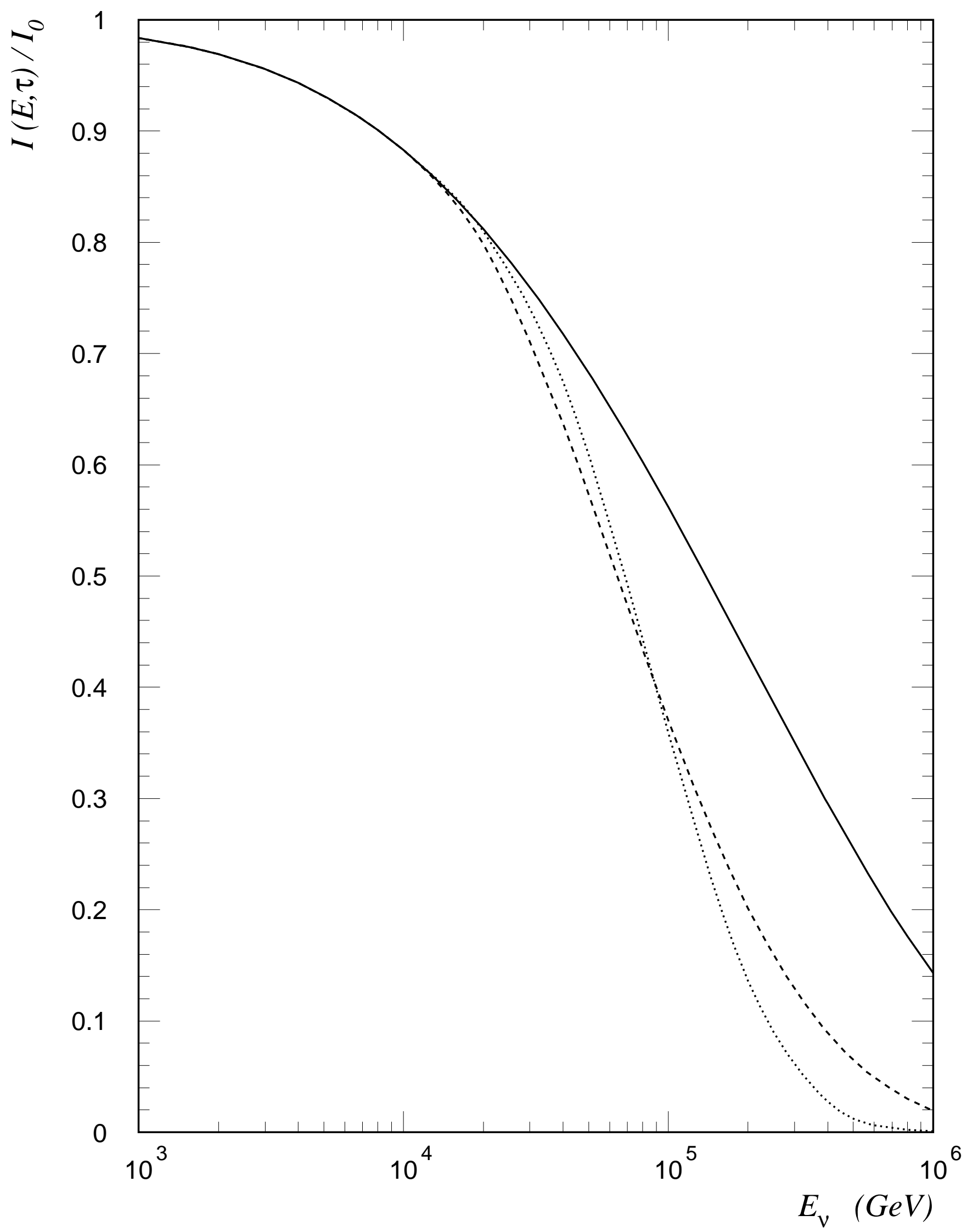

